\title{
Wissenschaft und Offenheit - Reflexion über Wissenschaft als Teil der Lehre zum wissenschaftlichen Arbeiten und Schreiben
}

Thomas Hapke

https://www.tub.tuhh.de/thomas-hapke/

hapke@tuhh.de,

https://blog.hapke.de/

https://orcid.org/0000-0002-5135-2693

2018, Preprint und unredigierte, etwas ausführlichere Manuskriptversion eines Beitrags zum "Praxishandbuch Schreiben in der Hochschulbibliothek", hrsg. von Willy Sühl-Strohmenger und Ladina Tschander (Berlin: De Gruyter 2019)

https://www.degruyter.com/view/product/501970

Publiziert über tub.dok, das Open Access Repository der Technischen Universität Hamburg (TUHH): https://doi.org/10.15480/882.1759 


\section{Zum Einstieg}

"Wissenschaftliche Arbeit wird überwiegend von äußerst unterschiedlichen Gruppen von Akteuren betrieben - Forschern aus verschiedenen Disziplinen, Amateuren und Professionellen, Menschen und Tieren, Funktionären und Visionären. Vereinfacht gesagt ist wissenschaftliche Arbeit heterogen. Zugleich erfordert Wissenschaft Kooperation - um gemeinsame Übereinkünfte zu erzielen, für Verlässlichkeit auf allen Gebieten zu sorgen und Informationen zu sammeln, die zeit- und raumübergreifend und in allen lokalen Eventualitäten ihre Integrität bewahren. Dies führt zu einer zentralen Spannung in der Wissenschaft, die zwischen unterschiedlichen Perspektiven und dem Bedarf an verallgemeinerbaren Befunden entsteht."1

"Die beiden Zitzen der Wissenschaft sind Gelegenheitsdiebstahl und Bricolage."2

Dieses zweite, hier dem Wissenschaftsphilosophen und-soziologen Bruno Latour ohne weitere Quellenangabe zugeschriebene Zitat ist bei Latour in dieser Form (deutsche Übersetzung!) zu finden: "Nach dem berühmten und hier leicht abgewandelten Wort von Sully sind 'Plünderung und Bastelei die beiden Brüste der Wissenschaft'."3 Der französische Herzog von Sully, Maximilian von Béthune, (1560-1641), hatte Ackerbau und die Weidewirtschaft als die Brüste von Frankreich bezeichnet.4 In einer lange nach Sullys Tod erschienenen deutschen Publikation hieß es: "Einer von Sully Hauptgrundsätzen war, daß der Feldbau und die Viehzucht die beyden Brüste eines Staates sind."5

1 Star, Susan Leigh u. James R. Griesemer: Institutionelle Ökologie, ı̈̈bersetzungenı und Grenzobjekte. Amateure und Professionelle im Museum of Vertebrate Zoology in Berkeley, 1907-39 (1989). In: Grenzobjekte und Medienforschung. (hg. von Sebastian Gießmann und Nadine Taha). Bielefeld: transcript Verlag 2017. 81-115, 81 Open Access unter http://oapen.org/search?identifier=640433.

2 Star, Susan Leigh: Grenzobjekte und Medienforschung. (hg. von Sebastian Gießmann und Nadine Taha). Bielefeld: transcript Verlag 2017, S. 428.

3 Latour, Bruno: Das Parlament der Dinge. Für eine politische Ökologie. 3. Aufl. Frankfurt am Main: Suhrkamp 2015, S. 20.

4 Wikipedia: Maximilien de Béthune, duc de Sully -- Wikipedia, Die freie Enzyklopädie. https:// de.wikipedia.org/w/index.php?title=Maximilien_de_B\%C3\%A9thune,_duc_de_ Sully\&oldid=178841262 (19.9.2018).

5 Sully, Maximilien de Béthune: Esprit de Sully. Oder: Auszug aus den Nachrichten des Herzogs von Sully, vornehmsten Ministers Heinrichs des Großen, von dem, was seine Verwaltung der Finanzen, und seine Grundsätze der Polizey betrifft : Nebst der Lobrede des Herrn Thomas auf den Herzog von Sully. Dresden: Gröll 1769, S. 527. https://books.google.de/books?id=IYCMS4hkajkC 
Abstract

Die Förderung wissenschaftlichen Arbeitens und Schreibens gehört im Rahmen von Informationskompetenz-Aktivitäten von Hochschulbibliotheken verstärkt zu deren Service-Portfolio. Die Tendenz zu Offenheit, zu Themen wie Open Access und Open Science, als aktuelle Herausforderung für die Wissenschaften betonen besonders Fragen von Wissenschaftlichkeit und die Qualität von Wissenschaft. Eine Auseinandersetzung damit kann auch die Lehre und das Lernen im Bereich des wissenschaftlichen Arbeitens und Schreibens befruchten. So wie zu einem ganzheitlichen Begriff von Informationskompetenz eine Reflexion über die Produktion von Information und ihren Medien gehört, so umfasst eine kritische Sicht auf wissenschaftliches Arbeiten als Teil von Bildung ein grundlegendes Verständnis des Funktionierens von Wissenschaft, ein Verständnis darüber, wie die unterschiedlichen Wissenschaften zu ihren Erkenntnissen und Ergebnissen kommen. Eine kritische Wissenschaftlichkeits-Kompetenz hat auch große Bedeutung für die aktuelle politische Diskussion um Fake News und für eine immer notwendiger werdende ökologische Kompetenz. Sie erleichtert in unserer vom wissenschaftlich-technischen Fortschritt und der Digitalisierung geprägten Gesellschaft wissenschaftlich begründete Entscheidungen im Alltag, etwa zum Umgang mit Lebensmitteln oder in der Gesundheits-Vorsorge, und in der Politik, etwa bezüglich der Umweltproblematik und des Nachhaltigkeits-Imperativs.

Fostering academic research and writing is more and more part of the services of university libraries within their activities to promote information literacy. The actual challenge and trend to openness, to topics like Open Access and Open Science, emphasizes especially questions of scientificality and the quality of science. A debate on this can enrich learning and teaching academic research and writing. As a holistic view on information literacy includes reflection about the production of information and its media, a critical view on academic research and writing comprehends an essential understanding of the functioning of scholarship and the sciences as part of education, an understanding, how the different sciences and disciplines arrive at their knowledge, insights and findings. A critical competence of scientificality is of great importance also for actual political discussions about fake news and for ecological literacy becoming essential more and more. In our society driven by scientific and technical progress and by digitalization, this competence facilitates decisions grounded on scholarly and scientific arguments and research in everyday's life (e.g. for health protection or when handling food) and in politics - for example concerning the environmental challenges (like the climate change) for a desirable sustainable future. 


\title{
1. Bibliotheken, Informationskompetenz und wissenschaftliches Arbeiten
}

\author{
„information literacy [and the practice of academic writing] is the practice of social \\ epistemology"6
}

Wissenschaftliches Arbeiten und forschendes Lernen beginnen für Forschende und Lernende auch in der Bibliothek. Dienstleistungen zur Unterstützung von Forschung und Lehre sind eine Kernaufgabe wissenschaftlicher Bibliotheken, deren Aufgaben sich durch die wachsende Digitalisierung, die laufenden und noch weiter zunehmenden Veränderungen bei der Hochschulbildung und beim wissenschaftlichen Publizieren weiter verändern werden.

Bibliotheken arbeiten verstärkt in Bereichen, die das gesamte Wissensmanagement der Universitäten betreffen. Aktivitäten im Bereich Informationskompetenz und wissenschaftliches Arbeiten, zum Aufbau eines Forschungsinformationssystems, die aktive Sichtbarmachung von Forschungsergebnissen in Open Access Repositories sowie Beratung zum Publizieren, zu Open Access, zu Forschungsdaten und zum Urheberrecht, aber auch Services im Rahmen von Projekten zur Öffnung der Lehre und zur Förderung von offener Wissenschaft („open science“), zeigen, dass forschungs- sowie lern- und lehrorientierte bibliothekarische Dienstleistungen immer mehr zusammenwachsen. Grundlegende angestrebte Tugenden oder Qualitäten, die das Handeln von Bibliotheken bestimmen, ähneln Werten, die für pädagogische Aktivitäten und auch für Forschungsund Schreibprozesse genannt werden: „critical thinking, collaboration, production, and openness".7

Lernangebote und Tutorials zum wissenschaftlichen Arbeiten und Schreiben, angeboten auch von wissenschaftlichen Bibliotheken, bieten im Optimum nicht nur Tipps und Rezepte sondern regen zum Nachdenken an, über das, was man selbst tut, die Auswirkungen der eigenen Tätigkeit im Kontext wissenschaftlichen Arbeitens und Schreibens, die Reflexion über das, was Wissenschaft kennzeichnet und wie wissenschaftliches Wissen entsteht. 8 Das wirkliche Erlernen wissenschaftlichen Arbeitens

6 Peter Tagtmeyer als Antwort auf eine Umfrage von Trudi E. Jacobson am 9. August 2016 in der Mailingliste ILI-L, vgl. ihre Zusammenstellung unter Finch, Janette, Van der Veer Martens, Betsy u.a.: Information Literacy Resources Suggested for Philosophy (from ILI-L). https://docs.google.com/ document/d/1fDgzL5Efca4nomnBhdup3WFtz19YY2ICQaA1ppWL4i0/edit (10.8.2018).

7 McCarthy, Seán u. Andrew Witmer: Notes toward a values-driven framework for Digital Humanities pedagogy. In: Hybrid pedagogy : a digital journal of learning, teaching and technology (2016) March 29, 2016.

8 Mit diesen Zielen beteiligt sich die Universitätsbibliothek der TUHH im Jahre 2018 mit dem Projekt "Wissenschaftliches Arbeiten öffnen" an der Hamburg Open Online University (HOOU), siehe https://www.tub.tuhh.de/wissenschaftliches-arbeiten/hoou-projekt-2018/, dies als Nachfolge zu einem Early-Bird-Projekt, vgl. Hapke, Thomas: Wie funktioniert eigentlich Forschung? In: HOOU Content Projekte der Vorprojektphase 2015/16 der Hamburg Open Online University. Hrsg. von Kerstin Mayrberger. Hamburg: Universität Hamburg 2017. S. 90-93. 
und Schreibens erfolgt in der Regel aber erst beim praktischen Handeln, etwa durch Publikationen von Studierenden als Lernprodukt via Open Access und/oder als Open Educational Resources (OER).9

Informationskompetenz wird zunehmend als Teil einer Kompetenz verstanden, die den gesamten Prozess wissenschaftlicher Kommunikation und des wissenschaftlichen Publizierens umfasst.10 Deutlich wird dies im amerikanischen „Framework for Information Literacy for Higher Education",11 in dem Wissenschaft als Konversation und Forschung als Prozess verstanden werden sowie Themen wie Autorität, Bewertung und Qualität von Wissenschaft eine Rolle spielen. So wie zu einem ganzheitlichen Begriff von Informationskompetenz eine Reflexion über die Produktion von Information und ihren Medien gehört, so ist für eine explizite Thematisierung wissenschaftlichen Arbeitens als Teil von Bildung ein Nachdenken über den Prozess bzw. die Produktion von Wissenschaft notwendig. Die Auseinandersetzung mit beiden im Rahmen der Lehre umfasst im Kern erkenntnistheoretische (epistemologische) Fragestellungen.12 Der bisher selten thematisierte Zusammenhang von Informationskompetenz mit "scientific literacy“ wird am Ende dieses Essay angesprochen.13

Die Argumentation erfolgt in vier Schritten: Zunächst wird hervorgehoben, dass die Tendenz zu Offenheit, zu Themen wie Open Access und Open Science, als aktuelle Herausforderung für die Wissenschaften besonders Fragen von Wissenschaftlichkeit und die Qualität von Wissenschaft betonen. Eine Auseinandersetzung damit kann die Lehre und das Lernen im Bereich des wissenschaftlichen Arbeitens und Schreibens befruchten. Nach einem Blick auf das Schreiben als Erkenntnisprozess wird für eine Meta-Sicht auf Wissenschaft hin zu einer kritischen Wissenschaftlichkeits-Kompetenz plädiert, die große Bedeutung für die aktuelle politische Diskussion um Fake-News und für eine immer notwendiger werdende ökologische Kompetenz haben kann.

9 Alexander, Laurie, Jason Colman u.a.: Publishing as Pedagogy: Connecting Library Services and Technology. EDUCAUSE Review. http://er.educause.edu/articles/2016/1/publishing-as-pedagogyconnecting-library-services-and-technology (10.8.2018); Einen ersten Versuch des Autors mit Studierenden: Hapke, Thomas (Hrsg.): Bibliothek in Zukunft?! Texte von Studierenden zum Wandel von Bibliotheken. Aus dem Bachelor-Seminar "Wissenschaftliches Arbeiten“ an der TU Hamburg. Hamburg: TUHH Universitätsbibliothek 2017.

10 Vgl. etwa Riehle, Catherine Fraser u. Merinda Kaye Hensley: What Do Undergraduate Students Know about Scholarly Communication? A Mixed Methods Study. In: portal: Libraries and the Academy 17 (2017) H. 1. S. 145-178.

$11 \mathrm{Vgl.} \mathrm{http://www.ala.org/acrl/standards/ilframework}$

12 Vgl. bzgl. Informationskompetenz auch Hapke, Thomas: Informationskompetenz anders denken zum epistemologischen Kern von „information literacy“. In: Handbuch Informationskompetenz. Hrsg. von Wilfried Sühl-Strohmenger. 2. Aufl. Berlin: De Gruyter Saur 2016. S. 9-21; Anderson, Anthony u. Bill Johnston: From information literacy to social epistemology. Insights from psychology. Cambridge, MA: Chandos 2016 sowie das Zitat am Beginn des Abschnittes.

$13 \mathrm{Vgl}$. Klucevsek, Kristin: The Intersection of Information and Science Literacy. In: Communications in Information Literacy 11 (2017) H. 2. S. 354-365. Dieses Essay geht aber über das in diesem Aufsatz geäußerte Verständnis einer wissenschaftlichen Kompetenz hinaus. 


\title{
2. Offenheit als aktuelle Herausforderung für die Wissenschaften
}

\author{
"Das eben haben wir mit Wissenschaft gemeint. Daß das Fragen wie das Antworten \\ mit Ungewißheit verbunden ist und daß beides weh tut. Doch daß es keinen Weg \\ drumherum gibt. Und daß man nichts verbirgt, sondern daß alles offen an Licht \\ kommt."14
}

Diese treffende und vielleicht auch überraschende Charakterisierung von Wissenschaft verbindet diese mit Unsicherheit und Ungewissheit, damit letztlich auch Wissenschaft mit Offenheit. Wissenschaftliches Arbeiten setzt eigentlich von Natur aus auf Offenheit des freien Wissensaustausches. Ein wichtiges Kennzeichen von Wissenschaft ist die Kommunikation. WissenschaftlerInnen, die ihre Forschungsergebnisse nicht publizieren und damit öffentlich machen, sind nicht wirklich Teil der weltweiten Wissenschaftsgemeinschaft.

Offenheit als Tugend ist Teil wissenschaftlicher Integrität, d.h. einer ethisch begründeten Haltung bzgl. des eigenen Handelns als Wissenschaftler. Offenheit soll Wissenschaft leichter zugänglich für alle machen. Nur Forschung, die an Offenheit orientiert ist, wirkt langfristig wirklich nachhaltig. Intersubjektivität, nach dem Wissenschaftsphilosophen Holm Tetens eines der fünf anzustrebenden Ideale von Wissenschaft,15 umfasst ein kooperatives, arbeitsteiliges Vorgehen, manchmal über mehrere Generationen hinweg und profitiert von Offenheit.

Digitalisierung und Open Science verändern das Verständnis von Wissenschaft in wissenschaftlichen Gemeinschaften und Wissenskulturen. Gerade Herausforderungen durch Open Access und Open Science bieten aktuelle Anknüpfungspunkte, um Wissenschaft als solche zum Thema zu machen. Lehrveranstaltungen zum wissenschaftlichen Arbeiten und Schreiben sind eine gute Möglichkeit, das Nachdenken über das Funktionieren von Wissenschaft, den Austausch über unterschiedliche Paradigmen in den Disziplinen, über Qualitätsmerkmale von Wissenschaften im Curriculum in allen Fächern zu verankern.

Die Wissenschaftshistorikerin Lorraine Daston beschreibt von ihr so genannte „moralische Ökonomien der Wissenschaft" als systemisch organisierte Gefüge von psychologisch und wertorientiert beeinflussten wissenschaftlichen Haltungen und Handlungen, etwa das Streben nach Quantifizierung, der Empirismus oder auch das, was man mit Objektivität bezeichnet.16 Diese Ökonomien sind historischem Wandel unterworfen. Zur moralischen Ökonomie des Empirismus gehört z.B. die Frage, was eine Tatsache ist. So wurden Tatsachen im 17. Jahrhundert anders gelebt

$14 \mathrm{H} \varnothing \mathrm{eg}$, Peter: Der Plan von der Abschaffung des Dunkels. Roman. Reinbek bei Hamburg: Rowohlt 1998, S. 25.

15 Vgl. Tetens, Holm: Wissenschaftstheorie. Eine Einführung. München: Beck 2013, $24 f f$ Die vier weiteren Ideale von Wissenschaft nach Tetens sind übrigens die Ideale der Wahrheit, der Begründung, der Erklärung und des Verstehens sowie der Selbstreflexion.

16 Daston, Lorraine: Die moralischen Ökonomien der Wissenschaft. In: Wunder, Beweise und Tatsachen. Zur Geschichte der Rationalität. 3. Aufl. Frankfurt am Main: Fischer Taschenbuch 2014. 157-184. 
und verstanden, als dies heute passiert, sie waren eher außergewöhnlich und singulär,17 etwa die Beschreibung eines Vulkanausbruches oder eines Erdbebens, und nicht etwas, was durch wiederhole Experimente „bewiesen“ worden war.

Daston zählt auch die Frage von Wissenschaft und Geheimhaltung zu den moralischen Ökonomien,18 so dass man auch Offenheit als eine solche beschreiben könnte. Offenheit als Tugend wissenschaftlichen Arbeitens - epistemische Tugenden, die Daston19 erwähnt, sind Objektivität,20 Wahrheit, Gewissheit und Genauigkeit - lässt sich historisch mindestens bis ins 17. Jahrhundert zurückverfolgen, wenn etwa Robert Boyle Offenheit wissenschaftlichen Wissens gegenüber Kritik fordert, das von anderen weltweit und zu jeder Zeit genutzt werden kann und was durch die Publikation von Ergebnissen gesichert werden sollte.21

Obwohl für Daston das Wort „Ökonomie" sich eher nicht auf wirtschaftliche Aspekte und die „Produktion und Distribution materieller Ressourcen“22 bezieht, ist für den französischen Philosophen Michel Serres „das erste Problem, das die Wissenschaft aufwirft, deren Verbreitung. Wenn finanzkräftige Unternehmen wissenschaftliche Ergebnisse aufkaufen, werden sie aus Eigeninteresse mit Blick auf einen möglichen Verkauf geheim gehalten und nur noch selektiv publiziert. Alles für Profit und Ruhm, [...] Wenn man ansieht, in welchem Maß Forschung von der Finanzierung abhängig geworden ist, welche Blüten die Publikationsbesessenheit treibt [...,] verbietet der berufliche Ehrenkodex eine Geheimhaltung, die auf kurz oder lang die gesamte Wissenschaft zersetzt und zerstört. Die egalitäre Veröffentlichung allen Wissens, die nichts zurückhält und nichts maßlos forciert und in den Vordergrund spielt, wird damit zur sittlichen Pflicht. Wissen muss öffentlich sein, davon ist nicht nur die Wissenschaft betroffen, sondern auch deren ethische Grundlage."23 Folgerichtig ist Open Access ebenfalls schon als moralische Ökonomie beschrieben worden.24

17 Ebd., 173.

18 Ebd., 177.

19 Daston, Lorraine: Wunder, Beweise und Tatsachen. Zur Geschichte der Rationalität. 3. Aufl. Frankfurt am Main: Fischer Taschenbuch 2014, S. 15.

20 Vgl. dazu Gaukroger, Stephen: Objektivität. Ein Problem und seine Karriere. Ditzingen: Reclam 2017; und besonders Daston, Lorraine u. Peter Galison: Objektivität. Frankfurt am Main: Suhrkamp 2007.

21 Neylon, Cameron: Openness in scholarship: A return to core values? In: Expanding perspectives on open science. Communities, cultures and diversity in concepts and practices : proceedings of the 21st International Conference on Electronic Publishing. Hrsg. von Leslie Chan u. Fernando Loizides. Amsterdam: IOS Press 2017. S. 6-17, S. 14.

22 Daston, Lorraine, 158.

23 Vorwort zu Serres, Michel u. Nayla Farouki (Hrsg.): Thesaurus der exakten Wissenschaften. Frankfurt am Main: Zweitausendeins 2001, S. 37-38.

24 Bacevic, Jana u. Chris Muellerleile: The moral economy of open access. In: European Journal of Social Theory 21 (2018) H. 2. S. 169-188. 
In den nächsten Abschnitten soll gezeigt werden, dass eine kritische Sicht auf den wissenschaftlichen Schreibprozess letztlich zu erkenntnistheoretischen und ebenso wie die Diskussion um Offenheit zu ethischen Fragestellungen führt und damit ein Nachdenken über Wissenschaft impliziert.

\title{
3. Schreiben als Erkenntnisprozess
}

\author{
"Keine Information ohne Transformation."25
}

„[... D]ie Last der Tradition, das Gewicht der Erziehung und die Wirkung der Reihenfolge des Erkennens. Dies sind soziale Momente und deshalb muß jede Erkenntnistheorie mit Sozialem und weiterhin mit Kulturhistorischem in Beziehung gebracht werden, insofern sie nicht in schwerem Widerspruch mit der Geschichte der Erkenntnis und der täglichen Erfahrung des Lehrenden und Lernenden geraten will."26

Schreiben ist letztlich Teil des Denkens, 27 es ist so etwas wie In-Form-Bringen des eigenen Denkens. Durch die Formulierung eigener Gedanken werden diese formiert bzw. formalisiert, andererseits wird dadurch das Denken trainiert. Beim Schreiben kommt man auf neue Gedanken und Assoziationen, es erleichtert zudem das Denken durch die Ablage und Speicherung von Gedanken. Schreiben ist "In-formation“ des Denkens, Information hier benutzt im Sinne einer seiner etymologischen Wurzeln als Einprägen bzw. Formen des Denkens, als "Bildung" (formation, engl.) des Denkens, im Sinne von Anregen des Denkens. Für den Philosophen Ernst Bloch hat jedes Erkennen zwei Seiten, ein Erkennen von Aspekten der Wirklichkeit und eine Veränderung, eine Formierung der Wirklichkeit.28 Dies ähnelt dem Eingangszitat dieses Abschnittes vom Wissenschaftsphilosophen und -soziologen Bruno Latour.

Schreiben ist also einerseits mehr als Aufschreiben, nicht nur Hilfsmittel und Werkzeug, nicht nur Darstellung und Repräsentation von Erforschtem oder Erkanntem sondern immer auch dessen Weiterentwicklung. Schreiben ist Teil des Erkenntnisprozesses, es schafft als Werkzeug ein Bild von Wirklichkeit, ordnet diese und formt diese damit auch. Auch der Wissenschaftssoziologe Ludwik

25 Latour, Bruno: Cogitamus. Berlin: Suhrkamp 2016 (Edition Unseld 38), S. 189.

26 Fleck, Ludwik: Zur Krise der »Wirklichkeit» (1929). In: Erfahrung und Tatsache. Gesammelte Aufsätze. Frankfurt am Main: Suhrkamp 1983. S. 46-58, S. 46.

27 Anregung für manche der folgenden Gedanken zu einer „Philosophie“ des wissenschaftlichen Schreibens fand der Autor dieses Essays bei Vorträgen von Bertrand Schütz, die dieser im Rahmen der "Kleinen Nacht des wissenschaftlichen Schreibens" an der TU Hamburg in den Jahren 2016 bis 2018 mit den Titeln „Sprache als Werkzeug", "Wissenskultur(en)" sowie „Schreiben und wissenschaftliche Praxis" gehalten hat.

28 Vgl. "Erkannt wird [...] zum Ziel der In-Formatio über die Welt und der Welt selber." Bloch, Ernst: Tübinger Einleitung in die Philosophie. Frankfurt am Main: Suhrkamp 1985, S. 44. 
Fleck formulierte in diesem Sinne: „Beobachten, Erkennen, ist immer ein Abtasten, also wörtlich Umformen des Erkenntnisgegenstandes.“29

Jede Überführung der Wirklichkeit in eine Form von Abbildung dieser, eine Repräsentation der Wirklichkeit, oft verbunden mit einer Abstraktion, lässt Teile dieser Wirklichkeit unberücksichtigt, betont andere usw., so dass man mit der gewonnenen Abbildung, die über die Wirklichkeit informieren soll, diese, die Wirklichkeit, auch transformiert. Die gewonnene Abbildung ist nun neu Teil der Wirklichkeit und kann selbst Grundlage weiterer Repräsentationen sein.

Andererseits verändert - ausgehend vom informiert-werdenden bzw. sich informierenden Individuum - jede Information das eigene Bewusstsein von einer Sache bzw. das eigene Wissen über diese. Bewusstsein oder Wissen werden also in einer andere "Form" transformiert. Schreiben als Beschreiben der eigenen experimentellen und praktischen Forschung umfasst auch eine neue Sicht auf diese, ein Neu-Entdecken des zum Beispiel im Experiment Erkanntem. Je mehr man sich mit einer Sache beschäftigt, desto mehr gewinnt diese für einen selbst an Komplexität, werden Verbindungen zu anderen Dingen sichtbar oder wird man auf Einflüsse aus vielfältigen Richtungen aufmerksam. Man erkennt, dass nichts so einfach ist, wie es am Anfang erscheint. Schreiben als Erkenntnisprozess hat den Charakter eines Experiments, ist offen für Überraschungen, da man dabei auch auf neue Gedanken kommt.

Wissenschaftliches Schreiben ist gleichzeitig als Prozess der Einführung in die Wissenskultur der jeweiligen Wissenschaft zu begreifen.30 Fängt man an wissenschaftlich zu schreiben, ist man eigentlich sofort dabei, auf die Schultern von Riesen31 zu steigen. Die gesamte bisherige Überlieferung der Wissenschaften ist latent und verborgen als Möglichkeit der Bezugnahme in Allem vorhanden, was man schreibt. Diese Latenz beim Schreiben kann am Anfang von Studierenden als große Last empfunden werden, von der man sich in einem gewissen Sinne auch freimachen muss, um als Subjekt eigene Gedanken denken zu können.32

Gerade Wissenschaftsphilosophen, die Wissenskulturen betrachten wie etwa Latour, Hans-Jörg Rheinberger und Karin Knorr Cetina, haben auf die Bedeutung der Praxis und des Experimentellen hingewiesen und ethnografische Forschungen zur Praxis durchgeführt, wobei das, was praktisch in der wissenschaftlichen Arbeit passiert, später durchaus anders dargestellt und kommuniziert werden kann, worauf auch Fleck hinweist: „Wenn von Naturwissenschaften die Rede ist, vergißt man meist,

29 Fleck, Ludwik (wie Anm. 26), S. 53.

30 Kruse, Otto: Wissenschaftliches Schreiben und studentisches Lernen. Zürich: Hochschuldidaktik UZH 2012, S. 7; Ethnografischen Forschungen zur Praxis wissenschaftlichen Arbeitens sind kürzlich auch in den Kulturwissenschaften durchgeführt worden: Krentel, Friedolin, Katja Barthel u.a. (Hrsg.): Library life. Werkstätten kulturwissenschaftlichen Forschens. Lüneburg: Meson Press 2015.

$31 \mathrm{Vgl}$. Hapke, Thomas: Auf den Schultern von Riesen. https://www.tub.tuhh.de/wissenschaftlichesarbeiten/2014/10/23/auf-den-schultern-von-riesen/ (10.8.2018).

32 Diesen Gedanken verdanke ich Bertrand Schütz. Latenz ist ebenso wie „Möglichkeit" auch ein Leitbegriff bei Ernst Bloch, vgl. Zeilinger, Doris: Latenz. In: Bloch-Wörterbuch. Leitbegriffe der Philosophie Ernst Blochs. Hrsg. von Beat Dietschy, Doris Zeilinger u. Rainer Zimmermann. Berlin, Boston: De Gruyter 2012. S. 232-242. 
daß es eine naturwissenschaftliche, lebendige Praxis gibt und parallel eine papierne, offizielle Gestalt."33

\title{
4. Von einer Meta-Sicht auf Wissenschaft zu einer kritischen Kompetenz der Wissenschaftlichkeit
}

\begin{abstract}
„Bildung besteht dann darin, einen Schritt hinter die begriffliche Routine zurückzutreten und sich auf einer zweiten Stufe der Aneignung zu fragen, wovon wir da eigentlich reden. [...] Was macht etwas zu einem Stück wissenschaftlichen Wissens? Und was ist das überhaupt: Wissenschaft? Woher rührt ihre Autorität? Zur Bildung gehört auch hier historisches Bewusstsein. Wie ist es zu einer Kultur gekommen, in der die Wissenschaft und ihre Technologie eine derart zentrale Rolle spielen?"34
\end{abstract}

„Was Wissenschaft vorführt, sind nicht alternativlose Fakten, sondern alternative Aussagen über jene Fakten, die Wissenschaft stets nur durch die Brille ihrer Theorien, Methoden und Verfahren sehen kann."35

Auf die Frage „Welche wissenschaftliche Idee ist reif für den Ruhestand?"36 antworteten 2014 wissenschaftliche Experten u.a. „Unsere enge Definition von Wissenschaft“ (Sam Harris), „Die Art und Weise, wie wir Wissenschaft produzieren und fördern“ (Kathryn Clancy), "Nur Wissenschaftler können Wissenschaft betreiben“ (Kate Mills), „Die wissenschaftliche Methode“ (Melanie Swan) oder "Gewissenheit. Absolute Wahrheit. Genauigkeit" (Richard Saul Wurman). Die Antworten zeigen, wie aktuell ein Nachdenken über Wissenschaft(en) und ihre Methoden ist.

Kern dieses Essays ist ein Plädoyer für eine Wissenschaftlichkeits-Kompetenz, eine Form von Bildung, um Wissenschaftlichkeit und das Funktionieren von Wissenschaft(en) zu verstehen. Bildung umfasst ein Nachdenken über Wissenschaft, die Reflexion über die eigene Disziplin und deren sozialgesellschaftliche Einbettung in die moderne Gesellschaft.37 Dazu gehören Fragen danach, welche Chancen aber auch welche Grenzen Wissenschaften bieten, welche unterschiedlichen Sichten auf Wissenschaft(en), welche unterschiedlichen Methoden möglich sind u.a. So wie man zur

33 Fleck, Ludwik (wie Anm. 26), S. 50.

34 Bieri, Peter: Wie wollen wir leben? 7. Aufl. München: dtv 2016, S. 68-69.

35 Nassehi, Armin: Die Rolle der Wissenschaften in der modernen Welt. Festvortrag im Rahmen der HRK-Jahresversammlung am 8. Mai 2017 in Bielefeld. Bonn: HRK 2017 (Beiträge zur Hochschulpolitik 2017,2), S. 6.

36 Brockman, John: Welche wissenschaftliche Idee ist reif für den Ruhestand? Die führenden Köpfe unserer Zeit über die Ideen, die uns am Fortschritt hindern. Frankfurt am Main: Fischer Taschenbuch 2016.

37 Vgl. das Zitat von Peter Bieri am Beginn dieses Abschnittes. 
„bewussten Förderung“ kritischen Denken dieses „selbst zur Sprache bringen“ sollte,38 ist für einen im Rahmen von Bildung notwendigen kritischen Blick auf Wissenschaft(en), die Wissenschaft selbst und ihr Anspruch an Wissenschaftlichkeit zur Sprache zu bringen. Kritisches Denken ist wesentlich „metatheoretisch“.39

Begründungen für ein explizites Nachdenken über Wissenschaft in der Bildung haben Tradition. Schon 1967 hieß es zu einem Symposium "Science and the human condition" in den Staaten: "The central problem of higher education is how to bring up new generations, fit to live as individuals and as citizens. The changing habitat which science is creating for them involves not only education in science, but perhaps even more importantly, education about science - the development of understanding of what science is about; what it can (and what it cannot) do, appreciation of the role of science in past history[,] of its likely role in the future [...]" 40

Der französische Soziologe Pierre Bourdieu machte 1986 im Rahmen des Collège de France Vorschläge für eine neue Bildung. Während einer seiner Vorträge sagte er, dass „die Wissenschaft in der gegenwärtigen Gesellschaft zu einem Machtinstrument geworden [sei ...], den Menschen müßten also ,Mittel zur Selbstverteidigung' an die Hand gegeben werden, mit denen sie sich gegen Manipulation aller Art wehren könnten. Wichtig sei hierfür, daß die Wissenschaften stets innerhalb ihres historischen Zusammenhangs gelehrt würden, damit die geschichtliche Bedingtheit der einzelnen Wissenschaften und auch des Rationalitätsbegriffs offensichtlich würde. Der Unterricht sollte interkulturell angelegt sein und die Pluralität der Kulturen betonen."41

Weltweite politische Entwicklungen machen es immer notwendiger, über die im Hintergrund stehende philosophische Frage nach dem Wesen von Wahrheit und Wissen, und damit von Wissenschaft nachzudenken. Die weltweite March-of-Science-Bewegung entstand als Antwort auf Tendenzen in Politik und Gesellschaft, die jeweilige Wirklichkeit nur noch populistisch oder als Fake News wahrzunehmen. Ein Verständnis von Wissenschaft kann selbst bei wissenschaftlich Arbeitenden recht einseitig sein, weisen doch manche der auf diesen zuerst im Frühjahr 2017

38 Kruse, Otto: Schreiben und kritisches Denken. Systematische und didaktische Verknüpfungen. In: Writing across the curriculum at work. Theorie, Praxis und Analyse. Hrsg. von Ursula Doleschal, Carmen Mertlitsch u.a. Wien: LIT 2013. S. 40-64, S. 40; vgl. zum kritischen Denken auch Kruse, Otto: Kritisches Denken und Argumentieren. Eine Einführung für Studierende. Konstanz, München: UVK/Lucius 2017.

39 Elkana, Yehuda u. Hannes Klöpper: Die Universität im 21. Jahrhundert. Für eine neue Einheit von Lehre, Forschung und Gesellschaft. Hamburg: Ed. Körber-Stiftung 2012, S. 211.

40 Rabinowitsch, Eugene: Science and the human condition. In: Bulletin of the Atomic Scientists 24 (1968) H. 8. S. 23. Die von Rabinowitsch herausgegebene Zeitschrift, das „Bulletin oft the Atomic Scientists", war von seiner Gründung 1945 an eine Zeitschrift, die die Wechselwirkungen zwischen Wissenschaft und Gesellschaft thematisierte, wobei in den 60er Jahren immer stärker auch die mit der Entwicklung von Wissenschaft und Technik einhergehenden Umweltbelastungen einen Platz fanden. Vgl. auch Hapke, Thomas: Kurz vor zwölf. https://www.tub.tuhh.de/blog/2016/04/14/kurzvor-zwoelf/ (1.10.2018)

41 Nolte, Dorothee: "Bildung als Selbstverteidigung". Der französische Soziologe Pierre Bourdieu zum Bildungswesen der Zukunft. In: Der Tagesspiegel 18.05.1986 (1986) H. 12356. S. 40; vgl. dazu Collège de France: Vorschläge für das Bildungswesen der Zukunft. In: Das Bildungswesen der Zukunft. Hrsg. von Sebastian Müller-Rolli. Stuttgart: Klett-Cotta 1987. S. 253-282. 
stattgefundenen Märsche gezeigten Plakate auf ein eher unkritisches Wissenschaftsverständnis hin, so als ob die Wissenschaft nur das Sagen haben müsste, damit wir in einer Welt voller Objektivität und Wahrheit leben können. Ein einfaches Plädoyer für die Berücksichtigung von Fakten aus der Wissenschaft in Politik und Gesellschaft übersieht, dass auch die Wissenschaften politischgesellschaftlichen Einflüssen unterliegen und dass Fakten nicht einfach so entstehen, sondern im Wissenschaftsprozess als " "Tat-Sachen" durchaus verhandelt und „mühsam erarbeitet werden. Die Wahrheit ist kein Fertighaus, das man über Nacht errichten kann."42

Außerdem gibt es nicht "die" Wissenschaft, sondern ganz viele Wissenschaften und unterschiedliche Sichtweisen, was Wissenschaft ist und wie diese funktioniert. Der Soziologe Armin Nassehi weist auf die Komplexität des Verhältnisses von Wissenschaft und Gesellschaft hin, die beide selbst voller Komplexität stecken. Er beschreibt Plakate mit der Aussage, dass es zu Fakten keine Alternative gäbe, als „Wissenschaftskitsch“.43 Manche Wissenschaftstreibenden haben bei ihrer Teilnahme interessanterweise den Aspekt der Bedeutung von "Open Science" in diesem Zusammenhang betont.44

Die Förderung von Informationskompetenz, die sich immer mehr vermischt mit Konzepten wie Datenkompetenz, einer "digital literacy“, „epistemic literacy“, "civic literacy“ oder "21st century skills“, verbunden mit einem Verständnis für das Funktionieren von Wissenschaft kann es erleichtern, Fake News zu erkennen. Fake News ist damit auch für Bibliotheken ein Thema.45 Jedoch gestalten Bibliotheken ihre Bildungsangebote im Bereich Informationskompetenz manchmal so, als ob es nur darum geht, in einem "one-stop shopping process" "solid nuggets of truth" anzubieten oder durch wissenschaftliches Arbeiten zu erreichen.46 Informationsquellen hinsichtlich Fake News kritisch zu bewerten ist ohne ein Verständnis darüber, wie (wissenschaftliches) Wissen entsteht und was unter Wahrheit verstanden werden kann, kaum möglich.

Offen bleibt diese Herausforderung: Wie lässt sich in der Gesellschaft und damit auch in der Bildung eine möglichst große Vielfalt und Diversität erreichen, wie lässt sich die immense Komplexität der Wirklichkeit, die ja unterschiedliche Sichten auf Wahrheit und Realität umfasst, sichtbar machen und abbilden, so dass man nicht so leicht auf einfache Antworten und Lösungen hereinfällt? Bei kaum einem Thema oder Problem gibt es heutzutage, wenn man genauer hinschaut und ggf. tiefer einsteigt, wirklich einfache Antworten und Lösungen. Latour betont etwa, dass "wir von Tag zu Tag deutlicher spüren, daß wir von der Unendlichkeit wieder zur Endlichkeit übergegangen sind oder vielmehr vom Unendlichen zum Vielfältigen, zum Komplizierten (compliqué), zum Verwickelten (impliqué)".47

42 Daston, Lorraine, Philipp Felsch u. Georg Mascolo: Welchen Fakten können wir trauen? In: Philosophie-Magazin (2017) H. 2. S. 58-63, S. 62.

43 Nassehi, Armin (wie Anm. 35), S. 6. Siehe auch das Zitat am Beginn dieses Abschnittes.

44 Brembs, Björn: Why I march. http://bjoern.brembs.net/2017/04/why-i-march/ (6.8.2018).

$45 \mathrm{Vgl}$. Anderson, Rick: Fake news and alternative facts: five challenges for academic libraries. In: Insights the UKSG journal 30 (2017) H. 2. S. 7-12 und den Blog der TIB Hannover https://blogs.tib.eu/wp/tib/tag/fake-news/.

46 Hicks, Alison: LibGuides: Pedagogy to Oppress? In: Hybrid pedagogy : a digital journal of learning, teaching and technology (2015) April 16.

47 Latour, Bruno (wie Anm. 25), S. 193. 
Der letzte Abschnitt gibt Hinweise zur genaueren thematischen Ausgestaltung einer Förderung von Wissenschaftlichkeits-Kompetenz, die neben erkenntnistheoretischen immer auch ethische Fragen berührt.

\title{
5. Wissenschafts-,„Innenpolitik“, -„Außenpolitik“ und ökologische Kompetenz
}

\author{
„[...] the research university, for which epistemology was always inextricable from
} ethics"48

„ecological literacy [...] is the ability to ask 'What then?"“49

Für eine Rückbesinnung auf wissenschaftliche Werte und Tugenden sowie eine Reflexion über die Bedeutung von Wissenschaft und Technik für das Überleben der Menschen auf der Erde sind Betrachtungen zur Vielfalt von Wissenschaft und deren Stellenwert im Rahmen von Wissenschaftsphilosophie, -theorie, -soziologie und -geschichte nützlich.50 Gesellschaftliche Aspekte wissenschaftlichen Arbeitens und Schreibens umfassen Themen, die implizit oder explizit im Rahmen diesbezüglicher Lehrveranstaltungen behandelt werden können, etwa zu Kontroversen in den Wissenschaften, oder zum Zusammenhang zwischen Wissenschaft und Demokratie.51 Thematische Aspekte einer Wissenschaftlichkeits-Kompetenz müssen sowohl Wissenschafts-„Innenpolitik" als auch Wissenschafts-„,Außenpolitik" im Fokus haben.

48 Wellmon, Chad: Organizing enlightenment. Information overload and the invention of the modern research university. Baltimore: Johns Hopkins University Press 2015, S. 8.

49 Ein Zitat von Garret Hardin in: Orr, David W.: Ecological literacy. Education and the transition to a postmodern world. Albany: State University of New York Press 1992, S. 85.

50 Beispiele mit teils populärgehaltenen, teils wissenschaftstheoretischem Hintergrund: Ahne, Verena u. Stefan Müller: Fast alles über Wissenschaft und Forschung. Wie Forschung funktioniert und was Wissenschaft eigentlich bedeutet. Wien: Verlag Holzhausen $\mathrm{GmbH}$ 2016; Holzer, Jacqueline, Jean-Paul Thommen u. Patricia Wolf: Wie Wissen entsteht. Eine Einführung in die Welt der Wissenschaft für Studierende und Führungskräfte. Zürich: Versus 2012; Tetens, Holm: Wissenschaftstheorie (wie Anm. 15); Ziche, Paul u. Joppe van Driel: Wissenschaft. http://nbnresolving.de/urn:nbn:de:0159-2011112141 (10.8.2018).

51 Vgl. Harker, David W.: Creating scientific controversies. Uncertainty and bias in science and society. Cambridge: Cambridge Univ. Press 2015; Leuschner, Anna: Die Glaubwürdigkeit der Wissenschaft. Eine wissenschafts- und erkenntnistheoretische Analyse am Beispiel der Klimaforschung. Bielefeld: Transcript 2012; Hagner, Michael: Wissenschaft und Demokratie. Berlin: Suhrkamp 2012 (Edition Unseld 47); Metschl, Ulrich: Vom Wert der Wissenschaft und vom Nutzen der Forschung. Zur gesellschaftlichen Rolle akademischer Wissenschaft. Wiesbaden: Springer VS 2016. 
Bildung für Wissenschaft fragt „innenpolitisch“, wie Wissenschaft eigentlich funktioniert, wie sie zu ihren Ergebnissen kommt, wie die Kommunikation, also auch das Publizieren, läuft, was etwa Peer Review ist, warum so selten negative Ergebnisse publiziert werden und anderes mehr. Historische Beispiele wie etwa die Kontroversen um den Sauerstoff in der Chemie Ende des 18. Jahrhunderts, die bis heute andauernden Diskussionen zur Evolutionstheorie Darwins und andere Themen mehr, bieten ausreichend Stoff, um Wissenschafts-, Innenpolitik“ zu verstehen. Die Untersuchung von Fallbeispielen, wo Wissenschaft Treibende gegen epistemische wissenschaftliche Tugenden, gegen die sogenannte "Guten wissenschaftlichen Praxis" verstoßen, die wissenschaftliche Integrität verletzen, z.B. bei Fälschungen in der Wissenschaft, sind sehr aufschlussreich zum Verstehen des Funktionierens von Wissenschaft und lehrreiche Beispiele für die Lehre des wissenschaftlichen Arbeitens und Schreibens.52

Der amerikanische Historiker Chad Wellmon betont, 53 dass das Erkennen in der Wissenschaft immer auch mit Ethik, also mit wertorientierten wissenschaftlichen Handlungen, verbunden ist. Gerade die institutionelle Entwicklung der Wissenschaft in Universitäten verbunden mit einer immer stärkeren Ausdifferenzierung der Disziplinen war, wie Wellmon zeigt, eine Antwort auf Fragen, welches Wissen wie legitimiert wird und eine Reaktion auf Entwicklungen des ausufernden Publikationswesens am Ende des 18. Jahrhunderts (,information overload“). Beschreibt Wellmon Herausforderungen an Wissenschaftlichkeit zur Wende zum 19. Jahrhundert, so hinterfragt der Wissenschaftshistoriker Paul Ziche das Verständnis von Wissenschaftlichkeit am Beginn des 20. Jahrhunderts im Rahmen pluralistischer „offener Wissenschaftslandschaften“.54 Die Themen an beiden Jahrhundertwenden wirken durchaus verwandt zu aktuellen Fragen zur Zukunft von Universitäten und des wissenschaftlichen Wissens, zur Frage eines pluralistischen, nichtreduktiven Verständnisses von Wissenschaft, ohne dass dieses in Relativismus abgleitet.

Ein „außenpolitischer" Blick macht bewusst, wie Wissenschaften und ihre Ergebnisse in Politik, Wirtschaft und Gesellschaft genutzt, kontrovers diskutiert und dabei nicht selten "falsch" interpretiert werden. So nutzen in der Klima- und Umweltpolitik unterschiedliche politische Lager und Interessengruppen Wissenschaft für die jeweils eigenen Zwecke. Wissenschaftliche Erkenntnisse werden bei Themen zur Gesundheit von Zigarettenkonsum, zum Klimawandel oder zum Einsatz von Glyphosat unterschiedlich als Argument verwendet, um Wissenschaft als Legitimation für die eigene Anschauung zu nutzen.

Latour rät Studierenden, zur Schärfung des Nachdenkens über die Wechselwirkung zwischen Wissenschaft, Politik und Gesellschaft, eine Art "Bordtagebuch"55 zu führen, um hier Ereignisse, Beispiele und deren Quellen (z.B. aus Tageszeitungen) festzuhalten. Daston weist auf die „formale Verwandtschaft der Tagebuchführung mit dem Anlegen von Experimentalprotokollen bzw.

52 Hapke, Thomas: Fake-News gibt es auch in der Wissenschaft. https://www.tub.tuhh.de/ wissenschaftliches-arbeiten/2018/07/30/fake-news-gibt-es-auch-in-der-wissenschaft/ (10.8.2018); vgl. auch Macrina, Francis L.: Scientific integrity. Text and cases in responsible conduct of research. 4. Aufl. Washington, DC: ASM Press 2014; und Finetti, Marco u. Armin Himmelrath: Der Sündenfall. Betrug und Fälschung in der deutschen Wissenschaft. Stuttgart: Raabe 1999.

53 Vgl. das Zitat am Beginn dieses Abschnittes.

54 Ziche, Paul: Wissenschaftslandschaften um 1900. Philosophie, die Wissenschaften und der nichtreduktive Szientismus. Zürich: Chronos 2008.

55 Latour (wie Anm. 25), S. 12. 
Feldbeobachtungen" hin.56 Solche Tagebücher könnten dann auch als Pool für Themen im Rahmen von Schreib-Projekten dienen. So wird sichtbar, wie sehr wissenschaftliche Ergebnisse Einfluss auf gesellschaftlich-politische Entscheidungen haben und umgekehrt, etwa bei der Problematik von Drittmittel- und Auftragsforschung oder beim Verhältnis zwischen Hochschulforschung und Militär, aber auch wie aktuell in den Vereinigten Staaten, dass wissenschaftliche Forschungsförderung durch den Staat teilweise in Frage gestellt wird.

Wissenschaftlichkeits-Kompetenz als wichtiger Teil von Allgemeinbildung ist zu unterscheiden von anderen Sichten auf wissenschaftliche oder akademische Kompetenz (,scientific literacy“). Gemeint ist nicht Kompetenz zum Erlernen wissenschaftlichen Arbeitens, um selbst wissenschaftlich tätig sein zu können. Die Ausbildung eines Verständnisses von Wissenschaft, um als Bürger sinnvoll mit Wissenschaft und Technik im Alltag umzugehen, passt eher. Als „critical scientific literacy “ meint Wissenschaftlichkeits-Kompetenz vor allem auch „öko-reflexive“ Bildung, um Wissenschaft kritisch zur Transformation in Richtung einer nachhaltigen Entwicklung in einer demokratischen und gerechten Gesellschaft zu nutzen.57

Die hier betonte Wissenschaftlichkeits-Kompetenz könnte die Herausforderung des Umgangs mit unserer Umwelt, mit dem nachhaltigen Erhalt unserer Lebensbedingungen wesentlich beeinflussen. Für individuelle und gesellschaftlich-politische Entscheidungen, die immer mehr durch Wissenschaften und deren Experten beeinflusst werden, von diesen in Frage gestellt aber auch dominiert werden können, ist ein Wissen über Wissenschaftsprozesse, Wechselwirkungen zwischen Wissenschaft und Gesellschaft und um die Problematik von Fake-News immer notwendiger. Nur wer wissenschaftliche Ergebnisse interpretieren kann, wird daraus verantwortungsvolle gesellschaftliche Schlussfolgerungen ziehen können.

"Verantworten" bedeutet auch "fähig sein zu antworten".58 Verantwortung für unsere Umwelt meint damit, beim Umgang mit der Umwelt Antworten zu finden und diese ins Handeln umzusetzen, damit eine lebenswürdige Umwelt erhalten bleibt. Verantworten bedeutet also auch, fähig sein zu handeln. Der sogenannte "ökologische Fußabdruck" (Ecological Footprint) stellt ein Maß für die Umweltbelastung durch einen Menschen bzw. den Verbrauch natürlicher Ressourcen durch einen Menschen dar. Die Umwelt- und Entwicklungsorganisation German Watch propagiert ergänzend im Rahmen einer "Bildung für eine nachhaltige Entwicklung" zum Erreichen der Zielen der Vereinten Nationen (Sustainable Development Goals) das Konzept des Handabdrucks („Hand Print“).59 Dieser impliziert, dass durch das "Hand"eln jedes einzelnen Menschen letztlich auch der ökologische Fußabdruck verringert werden kann. Vielleicht kann die Ausbildung einer Wissenschaftlichkeits-

56 Daston, Lorraine, 177.

57 Sjöström, Jesper u. Ingo Eilks: Reconsidering different visions of scientific literacy and science eduaction based on the concept of Bildung. In: Cognition, metacognition, and culture in STEM education. Learning, teaching and assessment. Hrsg. von Yehudit Judy Dori, Zemira R. Mevarech u. Dale R. Baker. Cham: Springer International Publishing 2018. S. 65-88, siehe Table 4.1, S. 78 und Fig. 4.2, S. 80 .

58 Bertrand Schütz in einem seiner Vorträge.

59 Reif, Alexander u. Marie Heitfeld: Wandel mit Hand und Fuß. Mit dem Germanwatch Hand Print den Wandel politisch wirksam gestalten. Bonn, Berlin: Germanwatch 2015. Online unter https://germanwatch.org/12040. 
Kompetenz den Handabdruck politisch Handelnder, sei es auf professioneller, ehrenamtlicher oder zivilgesellschaftlicher Ebene, deutlich erhöhen.

\section{Literaturverzeichnis}

Ahne, Verena u. Stefan Müller: Fast alles über Wissenschaft und Forschung. Wie Forschung funktioniert und was Wissenschaft eigentlich bedeutet. Wien: Verlag Holzhausen GmbH 2016.

Alexander, Laurie, Jason Colman u.a.: Publishing as Pedagogy: Connecting Library Services and Technology. EDUCAUSE Review. http://er.educause.edu/articles/2016/1/publishing-as-pedagogyconnecting-library-services-and-technology (10.8.2018).

Anderson, Anthony u. Bill Johnston: From information literacy to social epistemology. Insights from psychology. Cambridge, MA: Chandos 2016.

Anderson, Rick: Fake news and alternative facts: five challenges for academic libraries. In: Insights the UKSG journal 30 (2017) H. 2. S. 7-12.

Bacevic, Jana u. Chris Muellerleile: The moral economy of open access. In: European Journal of Social Theory 21 (2018) H. 2. S. 169-188.

Bieri, Peter: Wie wollen wir leben? 7. Aufl. München: dtv 2016.

Bloch, Ernst: Tübinger Einleitung in die Philosophie. Frankfurt am Main: Suhrkamp 1985.

Brembs, Björn: Why I march. http://bjoern.brembs.net/2017/04/why-i-march/ (6.8.2018).

Brockman, John: Welche wissenschaftliche Idee ist reif für den Ruhestand? Die führenden Köpfe unserer Zeit über die Ideen, die uns am Fortschritt hindern. Frankfurt am Main: Fischer Taschenbuch 2016.

Collège de France: Vorschläge für das Bildungswesen der Zukunft. In: Das Bildungswesen der Zukunft. Hrsg. von Sebastian Müller-Rolli. Stuttgart: Klett-Cotta 1987. S. 253-282.

Daston, Lorraine: Die moralischen Ökonomien der Wissenschaft. In: Wunder, Beweise und Tatsachen. Zur Geschichte der Rationalität. 3. Aufl. Frankfurt am Main: Fischer Taschenbuch 2014. 157-184.

Daston, Lorraine: Wunder, Beweise und Tatsachen. Zur Geschichte der Rationalität. 3. Aufl. Frankfurt am Main: Fischer Taschenbuch 2014.

Daston, Lorraine, Philipp Felsch u. Georg Mascolo: Welchen Fakten können wir trauen? In: Philosophie-Magazin (2017) H. 2. S. 58-63.

Daston, Lorraine u. Peter Galison: Objektivität. Frankfurt am Main: Suhrkamp 2007.

Elkana, Yehuda u. Hannes Klöpper: Die Universität im 21. Jahrhundert. Für eine neue Einheit von Lehre, Forschung und Gesellschaft. Hamburg: Ed. Körber-Stiftung 2012.

Finch, Janette, Van der Veer Martens, Betsy u.a.: Information Literacy Resources Suggested for Philosophy (from ILI-L). https://docs.google.com/document/d/ 1fDgzL5Efca4nomnBhdup3WFtz19YY2ICQaA1ppWL4i0/edit (10.8.2018).

Finetti, Marco u. Armin Himmelrath: Der Sündenfall. Betrug und Fälschung in der deutschen Wissenschaft. Stuttgart: Raabe 1999.

Fleck, Ludwik: Zur Krise der »Wirklichkeit» (1929). In: Erfahrung und Tatsache. Gesammelte Aufsätze. Frankfurt am Main: Suhrkamp 1983. S. 46-58.

Gaukroger, Stephen: Objektivität. Ein Problem und seine Karriere. Ditzingen: Reclam 2017. Hagner, Michael: Wissenschaft und Demokratie. Berlin: Suhrkamp 2012 (Edition Unseld 47). Hapke, Thomas: Auf den Schultern von Riesen. https://www.tub.tuhh.de/wissenschaftlichesarbeiten/2014/10/23/auf-den-schultern-von-riesen/ (10.8.2018). 
Hapke, Thomas: Informationskompetenz anders denken - zum epistemologischen Kern von „information literacy“. In: Handbuch Informationskompetenz. Hrsg. von Wilfried SühlStrohmenger. 2. Aufl. Berlin: De Gruyter Saur 2016. S. 9-21.

Hapke, Thomas: Kurz vor zwölf. https://www.tub.tuhh.de/blog/2016/04/14/kurz-vor-zwoelf/ (1.10.2018).

Hapke, Thomas (Hrsg.): Bibliothek in Zukunft?! Texte von Studierenden zum Wandel von Bibliotheken. Aus dem Bachelor-Seminar "Wissenschaftliches Arbeiten“ an der TU Hamburg. Hamburg: TUHH Universitätsbibliothek 2017.

Hapke, Thomas: Wie funktioniert eigentlich Forschung? In: HOOU Content Projekte der Vorprojektphase 2015/16 der Hamburg Open Online University. Hrsg. von Kerstin Mayrberger. Hamburg: Universität Hamburg 2017. S. 90-93.

Hapke, Thomas: Fake-News gibt es auch in der Wissenschaft. https://www.tub.tuhh.de/ wissenschaftliches-arbeiten/2018/07/30/fake-news-gibt-es-auch-in-der-wissenschaft/ (10.8.2018).

Harker, David W.: Creating scientific controversies. Uncertainty and bias in science and society. Cambridge: Cambridge Univ. Press 2015.

Hicks, Alison: LibGuides: Pedagogy to Oppress? In: Hybrid pedagogy : a digital journal of learning, teaching and technology (2015) April 16.

Høeg, Peter: Der Plan von der Abschaffung des Dunkels. Roman. Reinbek bei Hamburg: Rowohlt 1998.

Holzer, Jacqueline, Jean-Paul Thommen u. Patricia Wolf: Wie Wissen entsteht. Eine Einführung in die Welt der Wissenschaft für Studierende und Führungskräfte. Zürich: Versus 2012.

Klucevsek, Kristin: The Intersection of Information and Science Literacy. In: Communications in Information Literacy 11 (2017) H. 2. S. 354-365.

Krentel, Friedolin, Katja Barthel u.a. (Hrsg.): Library life. Werkstätten kulturwissenschaftlichen Forschens. Lüneburg: Meson Press 2015.

Kruse, Otto: Wissenschaftliches Schreiben und studentisches Lernen. Zürich: Hochschuldidaktik UZH 2012.

Kruse, Otto: Schreiben und kritisches Denken. Systematische und didaktische Verknüpfungen. In: Writing across the curriculum at work. Theorie, Praxis und Analyse. Hrsg. von Ursula Doleschal, Carmen Mertlitsch u.a. Wien: LIT 2013. S. 40-64.

Kruse, Otto: Kritisches Denken und Argumentieren. Eine Einführung für Studierende. Konstanz, München: UVK/Lucius 2017.

Latour, Bruno: Das Parlament der Dinge. Für eine politische Ökologie. 3. Aufl. Frankfurt am Main: Suhrkamp 2015.

Latour, Bruno: Cogitamus. Berlin: Suhrkamp 2016 (Edition Unseld 38).

Leuschner, Anna: Die Glaubwürdigkeit der Wissenschaft. Eine wissenschafts- und erkenntnistheoretische Analyse am Beispiel der Klimaforschung. Bielefeld: Transcript 2012.

Macrina, Francis L.: Scientific integrity. Text and cases in responsible conduct of research. 4. Aufl. Washington, DC: ASM Press 2014.

McCarthy, Seán u. Andrew Witmer: Notes toward a values-driven framework for Digital Humanities pedagogy. In: Hybrid pedagogy : a digital journal of learning, teaching and technology (2016) March 29, 2016.

Metschl, Ulrich: Vom Wert der Wissenschaft und vom Nutzen der Forschung. Zur gesellschaftlichen Rolle akademischer Wissenschaft. Wiesbaden: Springer VS 2016.

Nassehi, Armin: Die Rolle der Wissenschaften in der modernen Welt. Festvortrag im Rahmen der HRK-Jahresversammlung am 8. Mai 2017 in Bielefeld. Bonn: HRK 2017 (Beiträge zur Hochschulpolitik 2017,2). 
Neylon, Cameron: Openness in scholarship: A return to core values? In: Expanding perspectives on open science. Communities, cultures and diversity in concepts and practices : proceedings of the 21st International Conference on Electronic Publishing. Hrsg. von Leslie Chan u. Fernando Loizides. Amsterdam: IOS Press 2017. S. 6-17.

Nolte, Dorothee: "Bildung als Selbstverteidigung". Der französische Soziologe Pierre Bourdieu zum Bildungswesen der Zukunft. In: Der Tagesspiegel 18.05.1986 (1986) H. 12356. S. 40.

Orr, David W.: Ecological literacy. Education and the transition to a postmodern world. Albany: State University of New York Press 1992.

Rabinowitsch, Eugene: Science and the human condition. In: Bulletin of the Atomic Scientists 24 (1968) H. 8. S. 23.

Reif, Alexander u. Marie Heitfeld: Wandel mit Hand und Fuß. Mit dem Germanwatch Hand Print den Wandel politisch wirksam gestalten. Bonn, Berlin: Germanwatch 2015.

Riehle, Catherine Fraser u. Merinda Kaye Hensley: What Do Undergraduate Students Know about Scholarly Communication? A Mixed Methods Study. In: portal: Libraries and the Academy 17 (2017) H. 1. S. 145-178.

Serres, Michel u. Nayla Farouki (Hrsg.): Thesaurus der exakten Wissenschaften. Frankfurt am Main: Zweitausendeins 2001.

Sjöström, Jesper u. Ingo Eilks: Reconsidering different visions of scientific literacy and science eduaction based on the concept of Bildung. In: Cognition, metacognition, and culture in STEM education. Learning, teaching and assessment. Hrsg. von Yehudit Judy Dori, Zemira R. Mevarech u. Dale R. Baker. Cham: Springer International Publishing 2018. S. 65-88.

Star, Susan Leigh: Grenzobjekte und Medienforschung. (hg. von Sebastian Gießmann und Nadine Taha). Bielefeld: transcript Verlag 2017.

Star, Susan Leigh u. James R. Griesemer: Institutionelle Ökologie, ı̈̈bersetzungen und Grenzobjekte. Amateure und Professionelle im Museum of Vertebrate Zoology in Berkeley, 1907-39 (1989). In: Grenzobjekte und Medienforschung. (hg. von Sebastian Gießmann und Nadine Taha). Bielefeld: transcript Verlag 2017. 81-115.

Sully, Maximilien de Béthune: Esprit de Sully. Oder: Auszug aus den Nachrichten des Herzogs von Sully, vornehmsten Ministers Heinrichs des Großen, von dem, was seine Verwaltung der Finanzen, und seine Grundsätze der Polizey betrifft : Nebst der Lobrede des Herrn Thomas auf den Herzog von Sully. Dresden: Gröll 1769.

Tetens, Holm: Wissenschaftstheorie. Eine Einführung. München: Beck 2013.

Wellmon, Chad: Organizing enlightenment. Information overload and the invention of the modern research university. Baltimore: Johns Hopkins University Press 2015.

Wikipedia: Maximilien de Béthune, duc de Sully -- Wikipedia, Die freie Enzyklopädie. https:// de.wikipedia.org/w/index.php?title=Maximilien_de_B\%C3\%A9thune,_duc_de_ Sully\&oldid=178841262 (19.9.2018).

Zeilinger, Doris: Latenz. In: Bloch-Wörterbuch. Leitbegriffe der Philosophie Ernst Blochs. Hrsg. von Beat Dietschy, Doris Zeilinger u. Rainer Zimmermann. Berlin, Boston: De Gruyter 2012. S. 232242.

Ziche, Paul: Wissenschaftslandschaften um 1900. Philosophie, die Wissenschaften und der nichtreduktive Szientismus. Zürich: Chronos 2008.

Ziche, Paul u. Joppe van Driel: Wissenschaft. http://nbn-resolving.de/urn:nbn:de:0159-2011112141 (10.8.2018). 